\title{
Increased Metabolic Stress in Zucker Diabetic Fatty Rat Kidney and Pancreas
}

\author{
Haider Raza Annie John $^{\mathrm{a}}$ Frank Christopher Howarth ${ }^{\mathrm{b}}$ \\ Department of Biochemistry and Department of Physiology ${ }^{\mathrm{b}}$, College of Medicine and Health Sciences, \\ UAE University, Al Ain, United Arab Emirates
}

\section{Key Words}

Zucker diabetic rats $\bullet$ Pancreas $\bullet$ Kidney $\bullet$ Obesity $\bullet$ Oxidative stress $\bullet$ Mitochondrial dysfunction

\begin{abstract}
Background/Aims: Obesity and diabetes (hereafter termed diabesity) are among the most challenging global health problems. Since the main pathophysiological complications in diabesity are hyperglycemia, hyperlipidemia, insulin resistance, cardiomyopathy, nephropathy, and urinary infections, the kidney and pancreas are the potential target organs affected in the above conditions. However, the precise molecular mechanisms of disease progression and complications are still unclear. The Zucker homozygous (FA/FA) diabetic fatty (ZDF) rat is a genetic model for obesity and type 2 diabetes. Our previous studies, using cardiac muscles have demonstrated metabolic and oxidative stress in ZDF rats. In the present study, our aim was to investigate oxidative stress associated metabolic complications in ZDF rat kidney and pancreas. Methods: Here we have measured oxidative stress, glutathione (GSH)-dependent metabolism and mitochondrial respiratory functions in the kidney and pancreas of ZDF and Zucker lean $\left(Z L_{1}+/ F A\right)$ control rats. Results: Our results showed an increase in reactive oxygen species, NO production, lipid and protein peroxidation in ZDF rat kidney and pancreas accompanied by alterations in GSH-dependent metabolism and mitochondrial function. Western blot analysis has also confirmed increased expression of oxidative stress marker proteins in ZDF rats. Conclusion: We have demonstrated that ZDF rats develop metabolic complications associated with oxidative stress and mitochondrial dysfunction. Thus, these results might have implications in understanding the etiology and pathology of diabesity.
\end{abstract}




\section{Introduction}

The Zucker diabetic fatty (ZDF, FA/FA) rat is an experimental model of type 2 diabetes caused by an inherited insulin-resistant gene with progressive metabolic syndrome and hyperlipidemia [1, 2]. While male rats develop spontaneous diabetes and associated metabolic complications, female rats become diabetic only after intake of high fat diet. This selectivity is considered to be associated with decreased redox-homeostasis and enhanced insulin-resistance in male rats [2] when compared to female rats. The ZDF rats exhibit progressive cardiomyopathy, nephropathy and other cardiovascular and endothelial complications associated with increased oxidative and nitrosative stress [3, 4, 5]. ZDF rats with metabolic syndrome also display a cluster of different metabolic abnormalities such as obesity, hyperglycemia, hypertriglyceridemia, increased inflammatory biomarkers and oxidative stress and are considered one of the most appropriate experimental models of diabesity and insulin resistance [6]. Recent studies have also shown that inflammatory cytokines, excessive food intake and obesity are essential factors inducing hyperglycemia, hyperinsulinemia, insulin resistance and pancreatic $\beta$-cell dysfunction in ZDF rats when compared to Zucker FA/+ lean control rats [7]. The precise molecular mechanism of progressive metabolic complications and insulin resistance in ZDF rats is not completely understood. A recent study has shown that alteration in energy metabolism in progressive diabetes is correlated with increasing inflammation and oxidative stress [8]. These changes in energy metabolism and oxidative stress associated complications may have implications in understanding the etiology and pathophysiology of diabesity.

Our previous studies, using young (9-13 weeks) and elderly (30-34 weeks of age) ZDF rats $[9,10]$ reported differential cardiovascular responses to progressive hyperglycemia and metabolic complications in the young and elderly rats in comparison to lean controls. With age, the severity of diabetes and its complications worsens and ZDF rats are likely to become more reliant on the use of lipids and lipid reserves to meet metabolic requirements. This may partly account for the reduced weight in aged ZDF rats. A recent study by Daniels et al. [11] suggests that diabetes per se is not a critical factor in the induction of clinically significant cardiovascular complications and some other factors related to obesity might have greater impact in ZDF rats. Studies have also suggested that prolonged exposure to insulin suppresses the mitochondrial biogenesis and function and this may lead to impairment of insulin sensitivity $[12,13]$. Our recent study [14] using cardiac muscle from the left ventricle of ZDF rats has shown increased oxidative stress and mitochondrial dysfunction. Since the kidneys and pancreas are also known to be potential target organs affected in diabesity, we have extended our study to elucidate metabolic stress in these tissues. We have demonstrated that the metabolic complications in the kidney and pancreas of chronic diabetic ZDF rats are also associated with increased oxidative stress and mitochondrial dysfunctions.

\section{Materials and Methods}

\section{Chemicals}

Cytochrome c, reduced glutathione (GSH), oxidized glutathione (GSSG), 5,5'-dithio-bis(2-nitrobenzoic acid), 1-chloro 2,4-dinitrobenzene (CDNB), cumene hydroperoxide, dimethylnitrosamine (DMNA), dinitrophenylhydrazine (DNPH), glutathione reductase, thiobarbituric acid, NADH, NADPH, coenzyme Q2, antimycin A, dodecyl maltoside, and ATP Bioluminescent cell assay kits were purchased from SigmaAldrich Fine Chemicals (St Louis, MO, USA). 2', 7'- Dichlorofluorescein diacetate (DCFDA) was procured from Molecular Probes (Eugene, OR, USA). Polyclonal antibodies against CYP2E1, cytochrome c oxidase subunit 1, IKB- $\alpha$, TNF- $\alpha$, Nrf-2, iNOS, JNK and $\beta$-actin were purchased from Santa Cruz Biotechnology Inc. (Santa Cruz, CA, USA), GSH-Peroxidase from Abcam (Cambridge, MA, USA) and p-JNK from Cell Signaling Technology, Inc. (Danvers, MA, USA). Antibody for GSTA4-4 was a generous gift from Prof. Bengt Mannervik (Uppsala University, Uppsala, Sweden). Reagents for SDS-PAGE and Western blot analyses were purchased from Gibco BRL (Grand Island, NY, USA) and Bio Rad Laboratories (Richmond, CA, USA). 


\section{Animal and tissue preparation and sub-cellular fractionation}

Zucker diabetic fatty (ZDF; FA/FA) rats (average body wt. $=485 \mathrm{~g}$; average fasting blood glucose $=478$ $\mathrm{mg} / \mathrm{dl}$ ) and age-matched Zucker lean (ZL; +/FA) controls (average body wt. $=390 \mathrm{~g}$; average blood glucose $=108 \mathrm{mg} / \mathrm{dl}$ ) were purchased from Charles River Laboratories, UK as described in our previous study [14]. Approval for this project was obtained from the Animal Ethics Research Committee, College of Medicine \& Health Sciences, United Arab Emirates University and all the animals were used according to the safe practice for animals in research guidelines as stipulated by NIH, USA.

Kidneys and pancreas dissected from male ZDF and age-matched ZL control rats $(n=5)$ were rinsed with ice-cold saline and homogenized $(10 \% \mathrm{w} / \mathrm{v})$ in isotonic $100 \mathrm{mM}$ potassium phosphate buffer (pH7.4) containing $1 \mathrm{mM}$ EDTA and $0.1 \mathrm{mM}$ phenylmethylsulfonylfluoride (PMSF, a protease inhibitor). The homogenate was centrifuged at 1000xg for $10 \mathrm{~min}$ and the supernatant was used for further purification of mitochondrial fractions as described before [15]. Protein concentration in tissue homogenate and the isolated sub-cellular fractions was measured using BioRad reagent.

Measurement of ROS, NO and CYP $2 E 1$ activity

ROS production in ZDF and ZL rat kidney and pancreas was measured using the DCFDA fluorescence method as described before [16].

NO production was determined by Griess reagent as described in the vendor's protocol (R \&D Systems Inc). CYP 2E1-dependent N-demethylase activity was measured from the ZDF and ZL rat kidney and pancreas using dimethylnitrosamine (DMNA) substrate in the presence of NADPH in the appropriate buffer $(\mathrm{pH}$ 7.4) as described before [15].

Protein carbonylation and lipid peroxidation (LPO)

Protein peroxidation as a marker of increased oxidative stress was measured in ZDF and ZL rat tissue fractions by DNPH conjugation method as described before [17]. NADPH-dependent lipid peroxidation was measured as malonedialdehyde formed using the standard thiobarbituric acid method as described before [15].

Measurement of GSH-redox metabolism

GSH is the most important cellular antioxidant protecting tissues from oxidative insults. Alterations in GSH pools, GSH-redox metabolism by GSH-peroxidase/reductase and transferases are the key indicators of perturbed antioxidant metabolism. GSH concentration in the cytosol and mitochondria was measured by NADPH-dependent GSSG-reductase catalyzed conversion of GSSG to GSH. Glutathione S-transferase (GST) activity using CDNB, glutathione peroxidase (GSH-Px) activity using cumene hydroperoxide and glutathionereductase activity using GSSG/ NADPH as the respective substrates were measured by standard protocols as described before $[15,16]$.

Measurement of mitochondrial respiratory enzyme complex IV

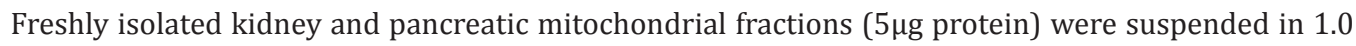
$\mathrm{ml}$ of $20 \mathrm{mM} \mathrm{KPi} \mathrm{buffer,} \mathrm{pH} 7.4$, in the presence of the detergent, lauryl maltoside (0.2\%). Cytochrome c oxidase (Complex IV) activity was measured using reduced cytochrome c, as a substrate according to the method of Birch-Machin and Turnbull, [18] as described previously [15].

\section{Measurement of ATP level}

ATP content in control ZL and ZDF rat tissue mitochondria was determined using ATP Bioluminescent cell assay kit (Sigma, St Louis, MO) according to the manufacturer's suggestion and samples were read using the TD-20/20 Luminometer (Turner Designs, Sunnyvale, CA).

\section{SDS-PAGE and Western blot analysis}

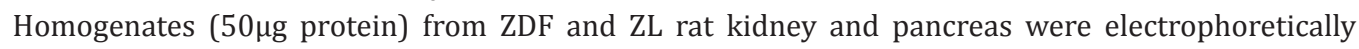
separated by $12 \%$ SDS-PAGE [19] and transferred on to nitrocellulose paper [20]. The expression of specific cell signaling and redox regulatory marker enzymes and proteins (iNOS, CYP2E1, GST A4-4, GSH-Px, Nrf-2, TNF- $\alpha$, Cyt c ox, JNK, p-JNK and IкB- $\alpha$ ) was checked by immunoreaction with their specific antibodies by Western blot analysis as described before [15]. Normalization of the protein loading was assessed using 


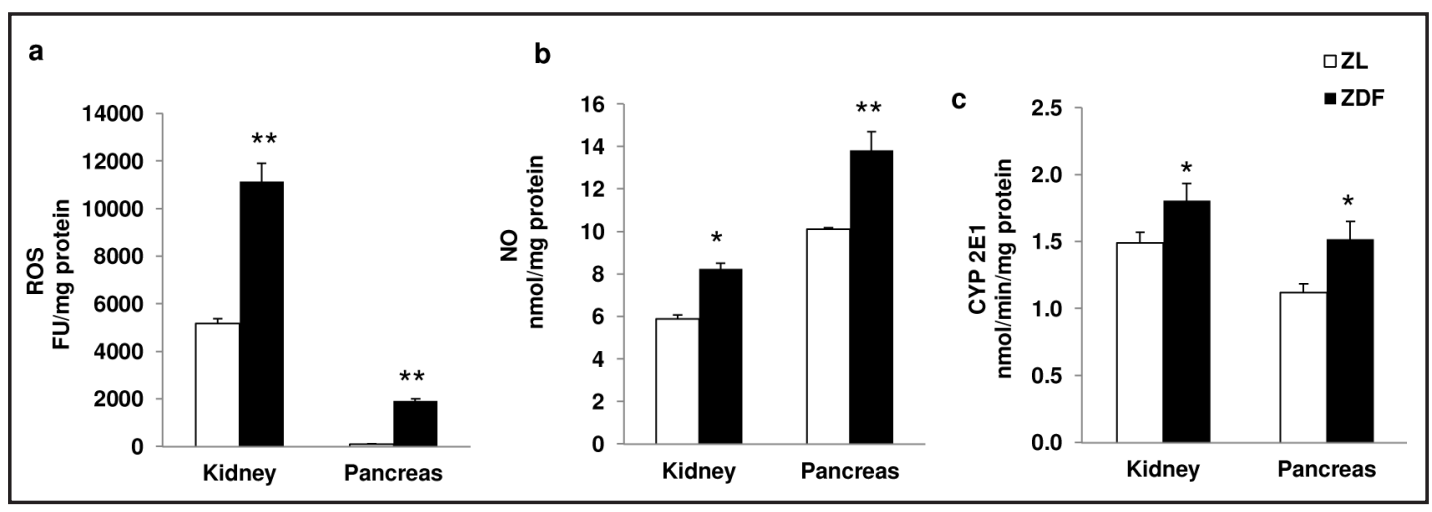

Fig. 1. ROS, NO production and CYP2E1 activity in ZDF rat kidney and pancreas. ROS production (a) in the kidney and pancreas of ZDF and ZL rats $(n=5)$ was measured using DCFDA as a probe as described in the Materials and Methods. Total homogenates from kidney and pancreas of ZDF and ZL rats were analyzed for total NO level (b) using Greiss reagent. CYP 2E1 activity (c) in ZDF and ZL rat tissues was measured using DMNA as substrate as described before [15]. Results are expressed as mean \pm S.E.M. from three independent experiments and asterisks indicate significant difference $\left({ }^{*} p<0.05\right.$ and $\left.{ }^{* *} p<0.01\right)$.

reversible Ponceau S staining and confirmed with $\beta$-actin as a loading control. Densitometry analysis of the protein bands was performed using a gel documentation system (Vilber Lourmat, France) and expressed as relative intensity (R.I) compared to the protein expression of control ZL which was arbitrarily taken as 1.0.

Data analysis

Values were calculated as mean \pm S.E.M. of at least three determinations. Statistical significance of the data was assessed using the unpaired' $t$ ' test for differences between groups and $p$ values $\leq 0.05$ were considered significant.

\section{Results}

\section{Sub-cellular oxidative stress and NO production}

There was a significant increase ( 2-fold) in plasma membrane bound NOX-dependent ROS formation (Fig. 1a) in the kidney and pancreas from ZDF compared to ZL control rats. Similarly, NO production was also significantly increased (36\% - 40\%) in the kidney and pancreas of ZDF rats when compared to ZL rat tissues (Fig. 1b).

A significant increase in CYP 2E1 activity was observed in the kidney and pancreas of ZDF compared to ZL control rats (Fig. 1c).

\section{Protein-lipid peroxidation}

Fig. 2a shows a three-fold increase in protein oxidative carbonylation as measured by DNPH-coupling in the kidneys and a 50\% increase in the pancreas of ZDF rats. A significant (40\% - 60\%) increase in LPO was observed in the kidneys and pancreas of the ZDF rats (Fig. $2 \mathrm{~b})$.

\section{Alterations in GSH content and metabolism}

A significant decrease in the total free GSH concentration was observed in the kidneys (30\%) and pancreas (20\%) (Fig.3a). The decrease in mitochondrial GSH was even more apparent in ZDF rat tissues as seen by a $40 \%$ decrease in GSH content in the kidney and pancreas when compared with ZL rat tissues (Fig. 3b). 


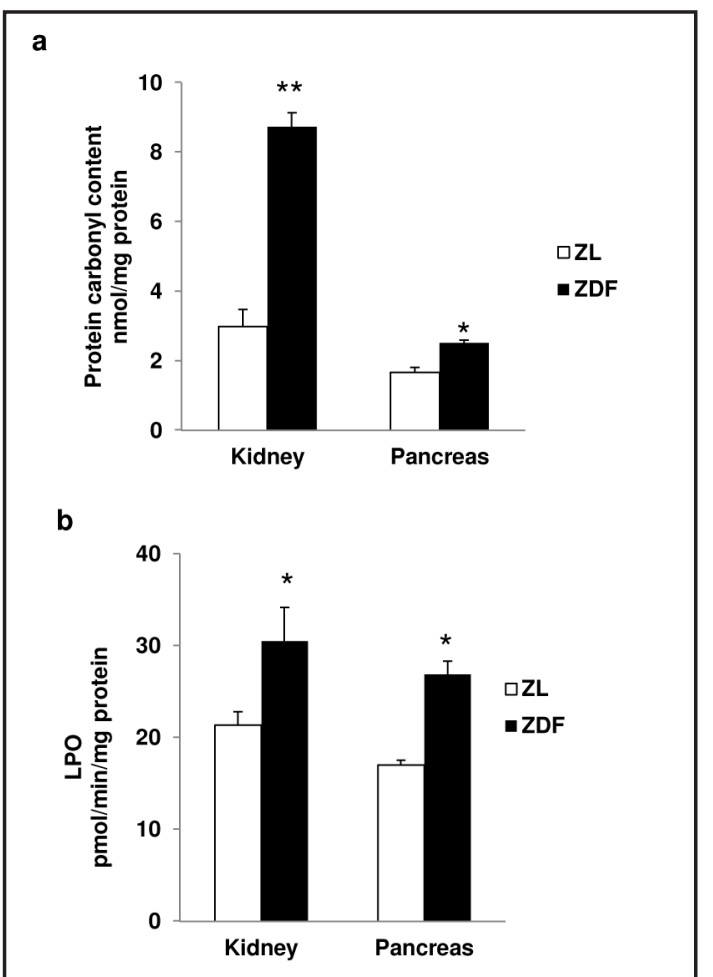

Fig. 2. Protein carbonylation and lipid peroxidation in ZDF rat kidney and pancreas. DNPH-coupled protein carbonylation (a) in the total homogenates from kidney and pancreas of ZDF and ZL rats $(n=5)$ was measured as described in the Materials and Methods. NADPH-dependent l LPO (b) was measured in the kidney and pancreas of ZDF and ZL rats as malonedialdehyde formed using the standard thiobarbituric acid method as described before [15].Results are expressed as mean \pm S.E.M. from three independent experiments and asterisks indicate significant difference $\left({ }^{*} \mathrm{p}<0.05\right.$ and $\left.^{* *} \mathrm{p}<0.01\right)$.

\section{a}

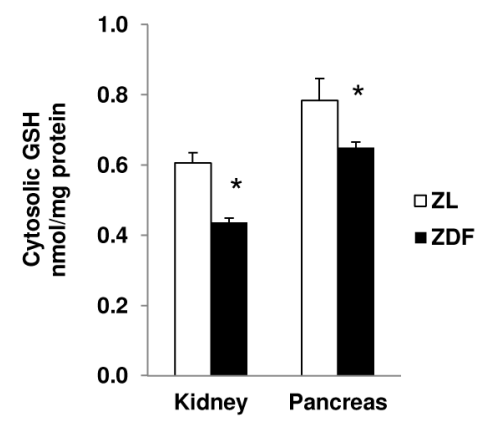

b

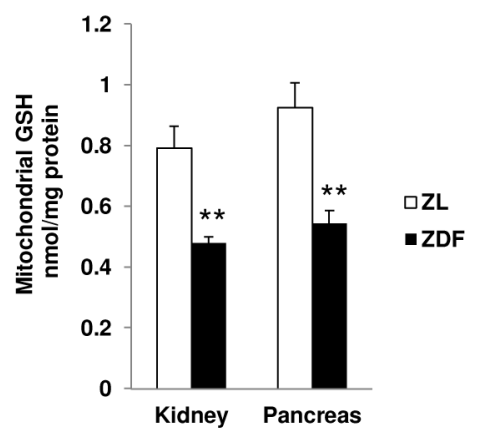

Fig. 3. GSH concentration in ZDF rat kidney and pancreas. GSH concentration in the kidney and pancreas of ZDF and ZL rats $(n=5)$ was measured in the cytosol (a) and mitochondria (b) by the enzymatic recycling method of Griffith as described in the Materials and Methods. Results are expressed as mean \pm S.E.M. from three independent experiments and asterisks indicate significant difference $\left({ }^{*} \mathrm{p}<0.05\right.$ and $\left.^{* *} \mathrm{p}<0.01\right)$.

Fig. 4. GSH-peroxidase activity in ZDF rat kidney and pancreas. GSH-Px activity in the kidney and pancreas of ZDF and ZL rats $(n=5)$ was measured in the cytosol (a) and mitochondria (b) using cumene hydroperoxide as a substrate was measured as described in the Materials and Methods. Results are expressed as mean \pm S.E.M. from three independent experiments and asterisks indicate significant difference $\left({ }^{*} p<0.05\right.$ and $\left.^{* *} p<0.01\right)$.

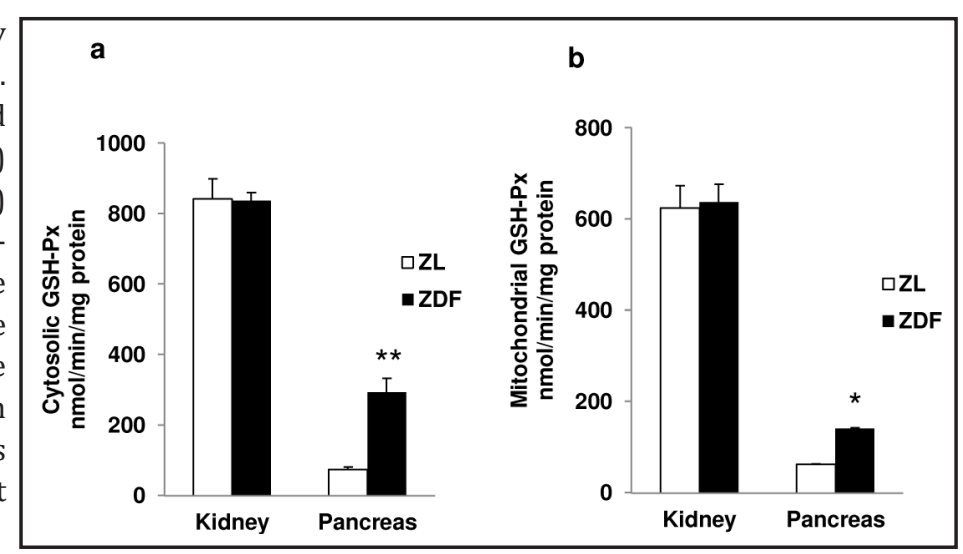

GSH-Px and GSH-Reductase activity

GSH-Px activity, showed differential responses in the kidney and pancreas of ZDF rats (Fig. 4a, b). While no appreciable effect was seen in cytosolic and mitochondrial GSH-Px 
Fig. 5. GSH-reductase activity in ZDF rat kidney and pancreas. GSHreductase activity in the kidney and pancreas of ZDF and ZL rats $(\mathrm{n}=5)$ was measured in the cytosol (a) and mitochondria (b) as described in the Materials and Methods. Results are expressed as mean \pm S.E.M. from three independent experiments and asterisks indicate significant difference $\left({ }^{*} \mathrm{p}<0.05\right.$ and ${ }^{* *} \mathrm{p}<0.01$ ).

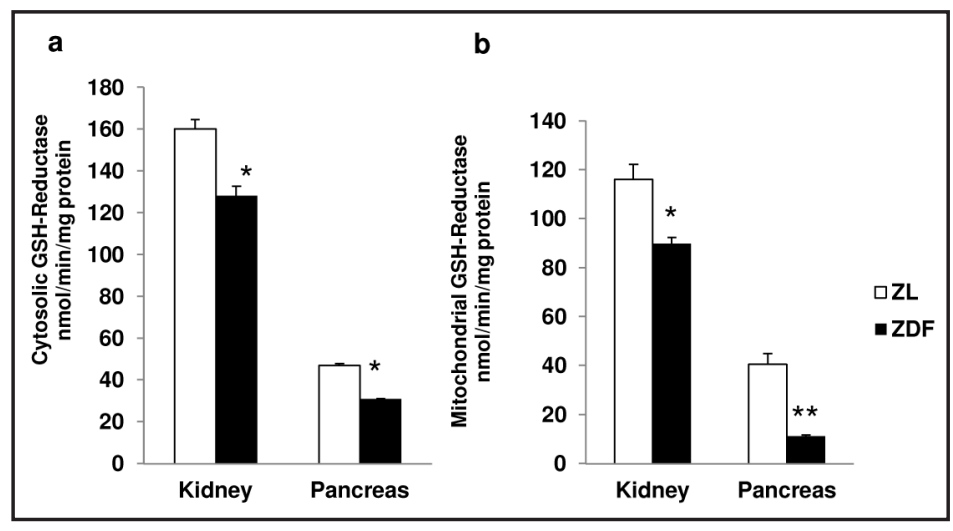

Fig. 6. GSH-conjugation activity in ZDF rat kidney and pancreas. GST activity in the kidney and pancreas of ZDF and ZL rats ( $n=5)$ was measured in the cytosol (a) and mitochondria (b) using CDNB as substrate as described in the Materials and Methods. Results are expressed as mean \pm S.E.M. from three independent experiments and asterisks indicate significant difference $\left({ }^{*} \mathrm{p}<0.05\right)$.

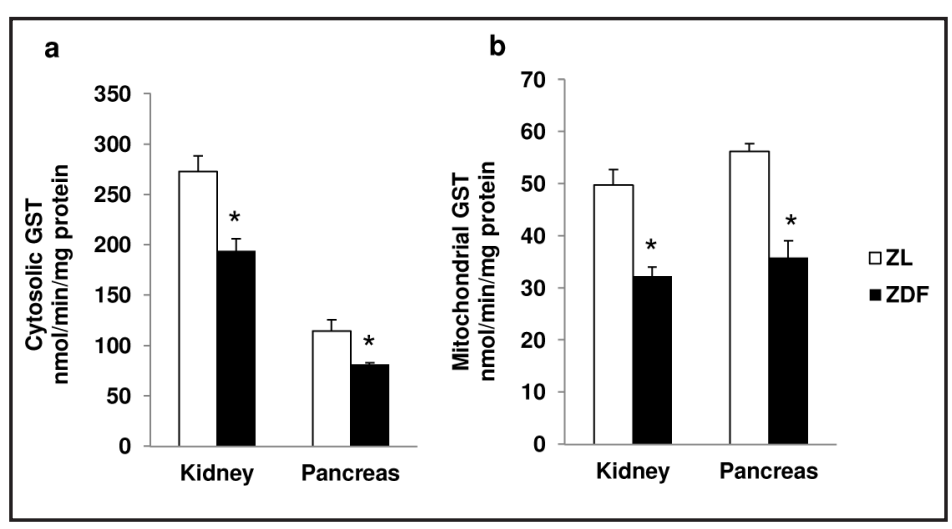

Fig. 7. Mitochondrial bioenergetics in ZDF rat kidney and pancreas. Mitochondrial respiratory enzyme Complex IV (a) activity in the kidney and pancreas of ZDF and $\mathrm{ZL}$ rats $(\mathrm{n}=5)$ was measured in freshly prepared mitochondrial fractions using reduced cytochrome $\mathrm{c}$ as a substrate as described in the Materials and Methods. The ATP content (b) in control ZL and ZDF rat tissues was determined using ATP Bioluminescent cell as-

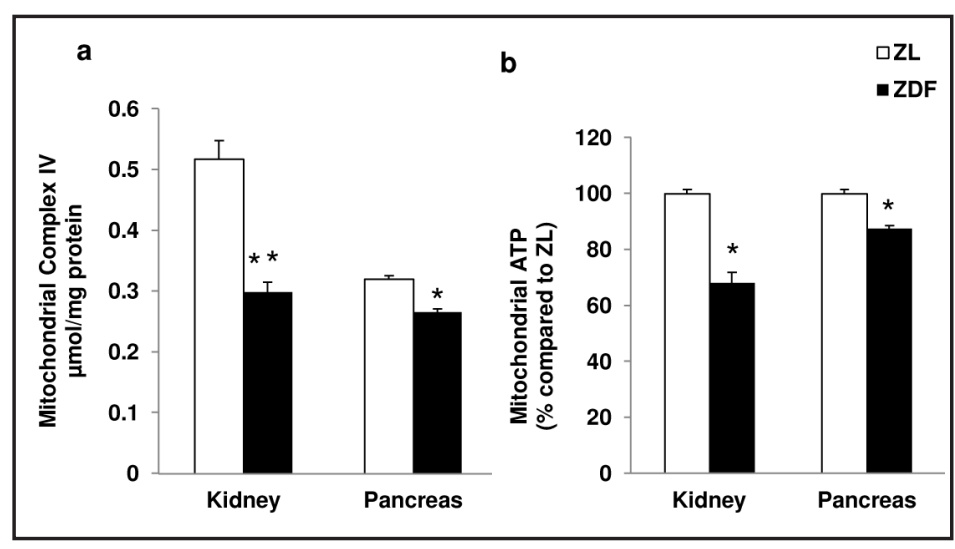
say kit (Sigma, St Louis, M0). Results are expressed as mean + S.E.M. from three independent experiments and asterisks indicate significant difference $\left({ }^{*} \mathrm{p}<0.05\right.$ and $\left.{ }^{* *} \mathrm{p}<0.01\right)$.

enzyme activity in the kidney, the enzyme activity increased significantly ( 2 to 4 -fold) in the pancreas. GSH- reductase activity, on the other hand was inhibited both in the kidney and pancreas from ZDF rats compared to ZL controls and the effect was more pronounced in the pancreas, when compared to the kidney (Fig. 5a, b). Total GSH-conjugating activity by GST, both in the kidney and pancreas from ZDF rat was significantly reduced in the cytosolic and mitochondrial fractions (Fig. 6a, b).

\section{Mitochondrial bioenergetics}

Figure 7 a shows a marked inhibition $(50 \%-60 \%)$ of mitochondrial respiratory Complex IV (cytochrome c oxidase) activity in the kidney from ZDF rats. Complex IV activity 


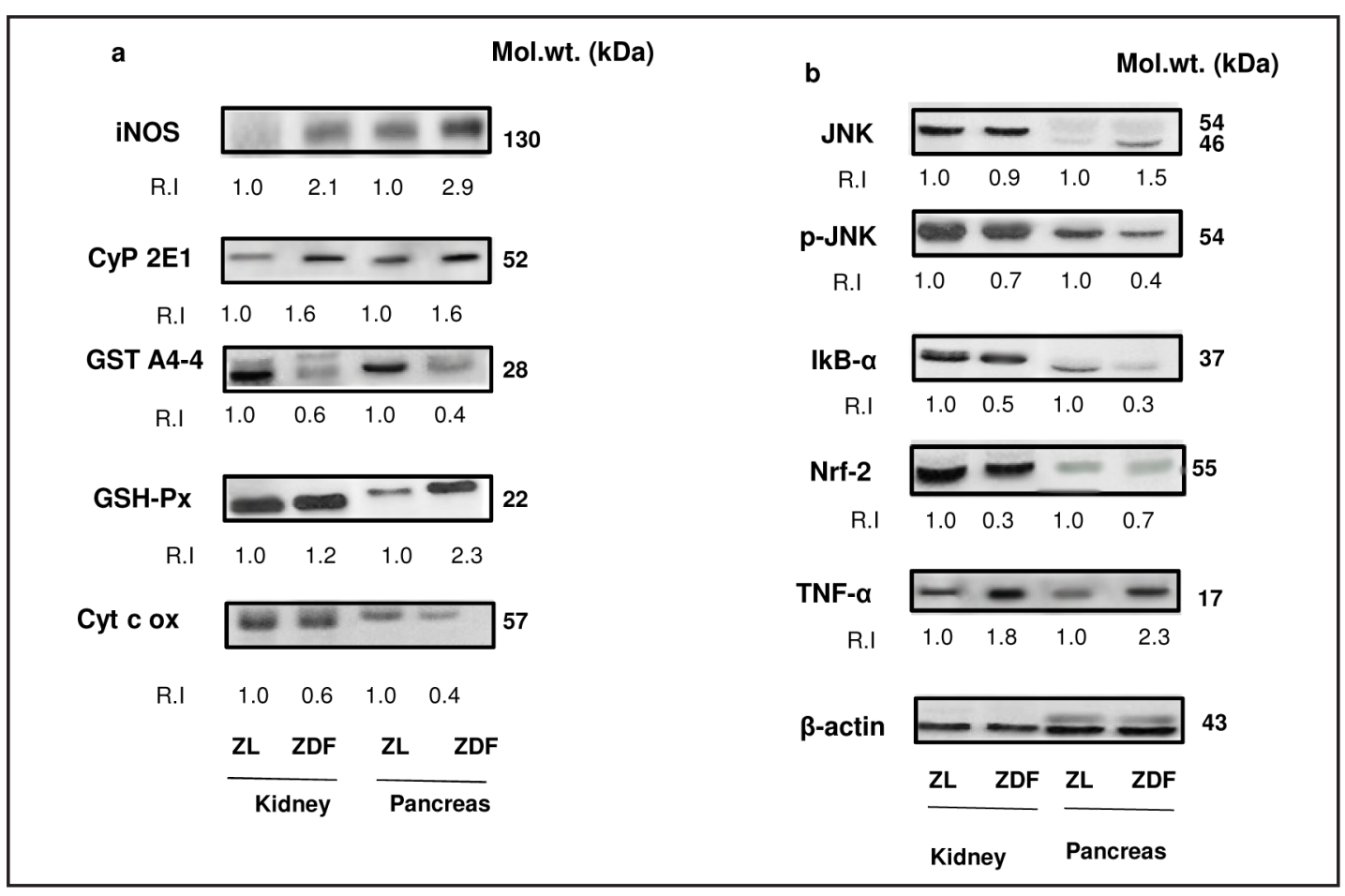

Fig. 8. Expression of redox-sensitive marker enzymes and proteins in ZDF rat kidney and pancreas. Representative samples from the kidney and pancreas ( $50 \mu \mathrm{g}$ protein) from ZDF and ZL rat were subjected to $12 \%$ SDS-PAGE and Western blot analyses individually, to visualize the expression of marker proteins using specific antibodies. (Fig.8a): iNOS, CYP2E1, GST A4-4, GSH-Px and Cyt c ox and (Fig.8b): JNK, p-JNK, IкB- $\alpha$, Nrf-2 and TNF- $\alpha$. Beta-actin was used as loading control. Relative intensity (R.I) of expressed proteins was calculated using the densitometer and normalized using the expression of the proteins in ZL as 1.0 as described before $[14,16]$. The figures shown are representative of 2-3 experiments. Molecular weights shown are in $\kappa$ Da.

was moderately, but significantly reduced in the pancreas as well. Similarly, ATP content was also significantly decreased in the kidney and moderately, but significantly decreased in the pancreas (Fig. 7b).

\section{Alterations in the expression of oxidative stress marker proteins}

Fig. 8 shows alterations in the expression of oxidative stress marker enzymes and proteins. The relative expression of iNOS, CYP2E1 and GSH-Px enzymes were increased in ZDF rats whereas a decreased expression of GSTA4-4 and mitochondrial Complex IV were observed (Fig.8a). These results are complementary to our observation on the alterations in catalytic activities of corresponding proteins. The expression of redox-sensitive proteins TNF- $\alpha$ and JNK (54/46 kDa) were relatively increased in ZDF rat tissues while a decreased expression of activated p-JNK, IкB- $\alpha$ and Nrf- 2 were observed .

\section{Discussion}

Our previous studies using both young and old ZDF rats have demonstrated metabolic and oxidative stress in isolated cardiac myocytes $[9,10,14]$. In the present study we have deliberately used older ZDF rats as they exhibit progressive metabolic complications and insulin resistance. Elderly rats ( $>22$ weeks old) exhibit multiple carbohydrate and lipid associated complications, such as hyperglycemia, hyperlipidemia and increased inflammatory 
biomarkers and cytokines $[2,4,5,21]$. Studies have also suggested that prolonged exposure to insulin suppresses mitochondrial function which may lead to impairment of insulin sensitivity and increased cytokine secretion $[12,13,22]$. Increased insulin resistance in type 2 diabetes has also been reported as a cellular defense mechanism against oxidative stressinduced mitochondrial dysfunction to protect cells from further oxidative damage [2325]. Increased ROS production is a common feature of insulin resistance and suppression of mitochondrial superoxide production increases insulin sensitivity and mitochondrial function [25]. Studies have shown an association of ROS production and inflammation in various organ dysfunctions. We have provided clear evidence of an increased oxidative stress and inflammatory response in ZDF rat tissues where increased ROS and NO production were accompanied by an increase in lipid peroxidation, protein carbonylation and alterations

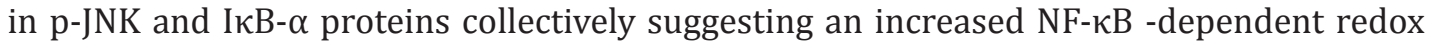
response. Increased expression of iNOS and CYP2E1 in our present study also suggests an increase in oxidative stress responses.

It is well known that reduced GSH protects cells against oxidative stress-induced toxicity and an increase in reduced GSH concentration is a defensive response against oxidative stress. Although the ratio of oxidized GSH and reduced GSH could not be measured in the tissues, our results clearly indicate a marked decrease in GSH concentration in ZDF rat pancreas which may be due to an alteration in GSH metabolism as shown by an increase in GSHperoxidase and a decrease in GSH- reductase activity. Western Blot analysis using specific antibody for GSH-Px showed a slight increase in the expression of the enzyme protein in the kidney while no significant alteration in the activity was observed. This may suggest the involvement and diversity of multiple GSH-metabolizing enzymes in the kidney as compared to the pancreas. Furthermore, various GST - isoenzymes, are also known to possess GSH-Px activity, in addition to GSH-conjugating activity, which may also contribute differentially to GSH-Px activity in the tissues. However, GSH conjugating activity by GST enzyme was also found to be reduced in ZDF rat kidney and pancreas, which was also confirmed by Western Blot analysis using antibodies against GSTA4-4 isoenzyme. Increased GSH-Px expression and activity in obese rats has been implicated in developing insulin resistance [26]. Lipid peroxidation product, 4-hydroxynonenal (4-HNE), can damage pancreatic $\beta$-cells and impair tissue response to insulin and thus plays a key role in developing metabolic syndrome [27]. Our results have also shown increased protein carbonylation and lipid peroxidation, accompanied by reduced GST activity and expression of 4-HNE metabolizing GST A4-4, suggesting increased accumulation of 4-HNE in ZDF rat kidneys and pancreas. There are reports suggesting that obese animals have low levels of GSH conjugating activity towards endogenous hydroperoxides, like 4-HNE, produced under oxidative stress conditions [28]. Our results also indicate increased ROS production in ZDF rat kidney and pancreas. CYP 2E1 activity, which is associated with ROS production and oxidative stress [29, 30], was also markedly increased in the kidney and pancreas.

Mitochondrial bioenergetics has also been affected in ZDF rat tissues. Our previous studies, using both type 1 and type 2 diabetic rat models, have suggested that increased oxidative stress induces mitochondrial dysfunction and compromises redox and respiratory metabolism in various tissues $[14,15,17]$. Our present results have also shown a reduction in mitochondrial bioenergetics as observed by reduction in ATP synthesis and cytochrome c oxidase activity.

In the present study, we have also demonstrated alterations in the expression of redox regulatory proteins, CYP 2E1, Nrf-2, IKB- $\alpha$ in ZDF rat kidney and pancreas. Increased CYP 2E1 and oxidative stress has been reported to activate Nrf-2 [29]. Hyperglycemia and oxidative stress has also been reported to be associated with altered NF- $\mathrm{\kappa B}$ signaling [31]. The increase in NF- $\kappa \mathrm{B}$ activation is associated with inhibition of $І \kappa \mathrm{B}$ phosphorylation. NF$\kappa B$ inhibition negatively regulates the activation of JNK [32]. Our results showed a decrease in the expression of activated p-JNK in the pancreas which is accompanied by an increased expression of JNK proteins. These observations were more apparent in the pancreas compared to the kidneys. Reduced expression of p-JNK in ZDF rat tissues has also been 
reported by others [7, 33]. Altered JNK-dependent signaling pathway, due to increased oxidative stress, has been reported in human and ZDF rat pancreas leading to the onset of mitochondrial dysfunction in the diabetic islets [34]. Our present study has also supported the above observation. Our previous study on cardiac muscles in ZDF rats has also reported a similar observation in the altered expression of JNK/p-JNK [14].

\section{Conclusion}

Our results on chronic diabetic ZDF rats have thus shown an increased expression of oxidative stress markers in the kidneys and pancreas. Mitochondrial respiratory functions and redox homeostasis were also affected in these tissues. Further studies on the role of altered signal transduction and antioxidant responsive transcription factors are needed to elucidate the molecular mechanism of progressive metabolic syndrome and insulin resistance in type 2 diabetic rats. Our results may help in better understanding the etiology and pathophysiology of diabesity and associated complications.

\section{Abbreviations}

CYP2E1 (cytochrome P450 2E1); DCFDA (2',7'-dichlorofluorescein diacetate); DMNA (dimethylnitrosamine); GSH (glutathione); GST (glutathione S-transferase); GSH-Px (glutathione peroxidase); GSH-reductase (glutathione reductase); Cyt c ox (cytochrome c oxidase); LPO (lipid peroxidation); ROS (reactive oxygen species); SDS-PAGE (sodium dodecylsulphate polyacrylamide gel electrophoresis); ZDF (Zucker diabetic fatty) ; ZL (Zucker lean).

\section{Conflict of Interest}

No conflict of interest to disclose for any of the authors

\section{Acknowledgements}

We acknowledge the financial research supports from Sheikh Hamdan Bin Rashid AlMaktoum Award for Medical Sciences, Terry Fox Cancer Research Fund and a grant from Research Committee, College of Medicine and Health Sciences, UAE University. Technical support from Ms Jasmin Shafarin is also acknowledged.

\section{References}

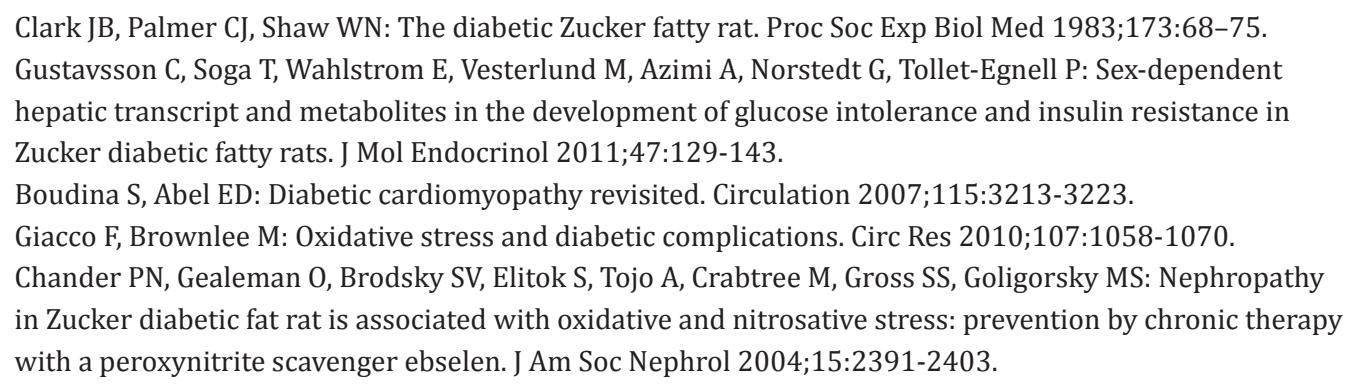




\section{Cellular Physiology $\quad$ Cell Physiol Biochem 2013;32:1610-1620 and Biochemistry

6 Sánchez D, Quiñones M, Moulay L, Muguerza B, Miguel M, Aleixandre A: Soluble fiber-enriched diets improve inflammation and oxidative stress biomarkers in Zucker fatty rats. Pharmacol Res 2011;64:31-35.

7 Chentouf M, Dubois G, Jahannaut C, Castex F, Lajoix AD, Gross R, Peraldi-Roux S: Excessive food intake, obesity and inflammation process in Zucker fa/fa rat pancreatic islets. PLoS ONE 2011;6:e22954.

-8 Liu TF, Vachharajani VT, Yoza BK, McCall CE: NAD-dependent sirt 1 and 6 proteins coordinate a switch from glucose to fatty acid oxidation during the acute inflammatory response. J Biol Chem 2012;287:2575825769.

-9 Howarth FC, Qureshi MA, Hassan Z, Al Kury LT, Isaev D, Parekh K, Yammahi SR, Oz M, Adrian TE, Adeghate E: Changing pattern of gene expression is associated with ventricular myocyte dysfunction and altered mechanisms of $\mathrm{Ca}^{2+}$ signalling in young type 2 Zucker diabetic fatty rat heart. Exp Physiol 2011;96:325337.

10 Howarth FC, Qureshi MA, Hassan Z, Isaev D, Parekh K, John A, Oz M, Raza H, Adeghate E, Adrian TE: Contractility of ventricular myocytes is well preserved despite altered mechanisms of $\mathrm{Ca}^{2+}$ transport and a changing pattern of mRNA in elderly type 2 Zucker diabetic fatty rat heart. Mol Cell Biochem 2012;361:267-80.

11 Daniels A, Linz D, van Bilsen M, Rutten H, Sadowski T, Ruf S, Juretschke HP, Neumann-Haefelin C, Munts C, van der Vusse GJ, van Nieuwenhoven FA: Long-term severe diabetes only leads to mild cardiac diastolic dysfunction in Zucker diabetic fatty rats. Eur J Heart Fail 2012;14:193-201.

12 Wang CH, Wang CC, Huang HC, Wei YH: Mitochondrial dysfunction leads to impairment of insulin sensitivity and adiponectin secretion in adipocytes. FEBS J 2013;280:1039-1050.

13 Liu H-Y, Yehuda-shnaidman E, Hong T, Han J, Pi J, Liu Z: Prolonged exposure to insulin suppresses mitochondrial production in primary hepatocytes. J Biol Chem 2009;284:14087-14095.

14 Raza H, John A, Howarth FC: Alterations in glutathione redox metabolism, oxidative stress, and mitochondrial function in the left ventricle of elderly Zucker diabetic fatty rat heart. Int J Mol Sci 2012;13:16241-16254.

15 Raza H, Prabu SK, Robin M-A, Avadhani NG: Elevated mitochondrial cytochrome P4502E1 and glutathione S-transferase A4-4 in streptozotocin-induced diabetic rats. Tissue specific variations and roles in oxidative stress. Diabetes 2004;53:185-194.

16 Raza H, John A, Nemmar A: Short-term effects of nose-only cigarette smoke exposure on glutathione redox homeostasis, cytochrome P450 1A1/2 and respiratory enzyme activities in mice tissues. Cell Physiol Biochem 2013;31:683-692.

17 Raza H, Prabu SK, John A, Avadhani NG: Impaired mitochondrial respiratory functions and oxidative stress in streptozotocin-induced diabetic rats. Int J Mol Sci 2011;12:3133-3147.

18 Birch-Machin MA, Turnbull DM: Assaying mitochondrial respiratory complex activity in mitochondria isolated from human cells and tissues. Methods Cell Biol 2001;65:97-117.

19 Laemmli UK: Cleavage of structural proteins during the assembly of the head of bacteriophage T4. Nature 1970;227:680-685.

20 Towbin H, Staehelin T, Gordon J: Electrophoretic transfer of proteins from polyacrylamide gels to nitrocellulose sheets: procedure and some applications. Proc Natl Acad Sci USA 1979;76:4350-4354.

-21 Shimabukuro M, Zhou YT, Levi M, Unger RH: Fatty acid-induced beta cell apoptosis: a link between obesity and diabetes. Proc Natl Acad Sci USA 1998;95:2498-2502.

22 Tianzheng Y, James LR, Yisang Y: Increased production of reactive oxygen species in hyperglycemic conditions requires dynamic change of mitochondrial morphology. Proc Natl Acad Sci USA 2006;103:26532658.

23 Tokuyama Y, Sturis J, DePaoli AM, Takeda J, Stoffel M, Tang J, Sun X, Polonsky KS, Bell GI: Evolution of betacell dysfunction in the male Zucker diabetic fatty rat. Diabetes 1995;44:1447-1457.

24 Shen GX: Oxidative stress and diabetic cardiovascular disorders: roles of mitochondria and NADPH oxidase. Can J Physiol Pharmacol 2010;88:241-248.

25 Hoehn KL, Salmon AB, Hohnen-Behrens C, Turner N, Hoy AJ, Maghzal GJ, Stocker R, Van Remmen H, Kraegen EW, Cooney GJ, Richardson AR, James DE: Insulin resistance is a cellular antioxidant defense mechanism. Proc Natl Acad Sci USA 2009;106:17787-17792.

26 McClung JP, Roneker CA, Mu W, Lisk DJ, Langlais P, Liu F, Lei XG: Development of insulin resistance and obesity in mice overexpressing cellular glutathione peroxidase. Proc Natl Acad Sci USA 2004;101:88528857. 
27 Mattson MP: Roles of the lipid peroxidation product 4-hydroxynonenal in obesity, the metabolic syndrome, and associated vascular and neurodegenerative disorders. Exp Gerentol 2009;44:625-633.

28 Singh SP, Niemczyk M, Saini D, Awasthi YC, Zimniak L, Zimniak P: Role of the electrophilic lipid peroxidation product 4-hydroxynonenal in the development and maintenance of obesity in mice. Biochemistry 2008;47:3900-3911.

29 Cederbaum AI: Cytochrome P450 2E1-dependent oxidant stress and upregulation of anti-oxidant defense in liver cells. J Gastroen Hepatol 2006;21:S22-S25.

30 Zhang W, Lu D, Dong W, Zhang L, Zhang X, Quan X, Ma C, Lian H, Zhang L: Expression of CYP2E1 increases oxidative stress and induces apoptosis of cardiomyocytes in transgenic mice. FEBS J 2011;278:1484-1492.

31 Tsai KH, Wang WJ, Lin CW, Pai P, Lai TY, Tsai CY, Kuo WW: NADPH oxidase-derived superoxide anioninduced apoptosis is mediated via the JNK-dependent activation of NF- $\kappa \mathrm{B}$ in cardiomyocytes exposed to high glucose. J Cell Physiol 2012;227:1347-1357.

32 Anning L: Activation of the JNK signaling pathway: breaking the brake on apoptosis. Bioessays 2002;25:1724.

33 Lakshmanan AP, Harima M, Sukumaran V, Soetikno V, Thandavarayan RA, Suzuki K, Kodama M, Nagata M, Takagi R, Watanabe K: Modulation of AT-1R/AMPK-MAPK cascade plays crucial role for the pathogenesis of diabetic cardiomyopathy in transgenic type 2 diabetic (Spontaneous Diabetic Torii) rats. Biochem Pharmacol 2012;83:653-660.

-34 Syed I, Kyathanahalli CN, Jayaram B, Govind S, Rhodes CJ, Kowluru RA, Kowluru A: Increased phagocytelike NADPH oxidase and ROS generation in type 2 diabetic ZDF rat and human islets: role of Rac1-JNK1/2 signaling pathway in mitochondrial dysregulation in the diabetic islet. Diabetes 2011;60:2843-2852. 